\title{
Can immature granulocytes indicate mortality in patients with acute ischemic stroke?
}

\author{
Mustafa Korkut, Cihan Bedel, Ökkeş Zortuk, Fatih Selvi \\ Department of Emergency Medicine, Health Science University, Antalya Training and Research \\ Hospital, Antalya, Turkey
}

\begin{abstract}
Background \& Objective: Stroke is the most common cause of permanent disability and the most important cause of mortality. Acute ischemic stroke (AIS) reveals inflammation in the ischemic brain tissue. Ischemic tissue causes proinflammatory cytokine release and aggregation of immune cells. Therefore in this study, we aimed to investigate the role of immature granulocyte (IG) in showing 30-day mortality in patients with AİS. Methods: This study was designed as a single-centered, retrospective cohort study. Patients aged $>18$ years who were diagnosed with AIS in the tertiary emergency department were included in this study. Patients were divided into two groups as low $(<0.6 \%)$ and high $(\geq 0.6 \%)$ by IG values. Demographic and laboratory parameters were compared between the groups at admission to the emergency department. Results: Our study consisted of 172 patients diagnosed with AIS, who met the inclusion criteria. The average age of the study group was $69.19 \pm 14.34$ years, and $94(54.7 \%)$ of the patients were male. $98(56.9 \%)$ patients were in the low IG group, and $74(43.1 \%)$ of them were in the poor outcome group. IG at the cut-off value of 1.3 was shown to predict mortality in patients with AIS with $80.5 \%$ sensitivity and $93.2 \%$ specificity (area under the curve: $0.71595 \%$ CI: $0.623-0.807, p<0.001$ )

Conclusion: The results of our study showed that IG is a new and simple predictor to predict 30-day prognosis in patients with AIS.
\end{abstract}

Keywords: Immature granulocytes, inflammation, acute ischemic stroke

\section{INTRODUCTION}

Stroke is the most common cause of permanent disability and the most important cause of mortality around the world and is defined as focal or global impairment of cerebral functions. Ischemic stroke is the most common type of stroke and is seen in 9 out of 10 stroke patients. ${ }^{1}$ Stroke is the second most common cause of mortality after coronary artery disease and malignancies, with an annual prevalence of around $6 / 1000{ }^{2}$ Therefore, it is important to understand the development mechanism of the disease and to make early prediction of mortality.

The role of inflammation in the pathophysiology of acute ischemic stroke (AIS) has been demonstrated by studies. ${ }^{3}$ Inflammatory mediators have been measured to be at lower levels in normal brain tissue. Ischemic tissue causes proinflammatory cytokine release and aggregation of immune cells. ${ }^{4}$ The relationship of poor prognosis with NLR (neutrophil-to-lymphocyte ratio), LMR (lymphocyte-to-monocyte ratio), PLR (platelet-to-lymphocyte ratio) that were obtained from white blood cell count in patients with AIS, as a marker of systemic inflammation, has been reported in previous studies. ${ }^{5-7}$ Immature granulocyte (IG) is a rapidly obtained parameter from an automated blood cell analyzer, and it has recently been shown to be useful in predicting the complications and mortality of many diseases such as sepsis, septic shock, local infection, acute appendicitis and pancreatitis. - $^{8-10}$

However, the role of IG in the 30-day mortality of patients with AIS remains unclear. Therefore, in this study, we wanted to evaluate the role of IG in showing 1-month mortality in patients with AIS.

\section{METHODS}

Our study was designed as a single-center retrospective cohort study. Patients were selected among the patients who admitted to the tertiary 
emergency department between January 1, 2019, and January 1,2020, and diagnosed with AIS and were confirmed to have acute infarction in brain computed tomography (CT) or magnetic resonance imaging (MRI). Our patient population consisted of all patients with AIS in brain CT or diffusionweighted MRI taken in the emergency department. Exclusion criteria were determined as all patients $<18$ years of age, pregnant women, patients with a disease or drug use that could change laboratory parameters, traumatic intracranial hemorrhages, patients with 24 hours after from the onset of symptoms, abnormal variations in MRI/CT, and patients with incomplete data. Infarct localization was checked from imaging reports. The Glasgow Coma Scale (GCS) values of the patients at admission to the emergency department and their vital signs at the time of admission were recorded. Demographic data of AIS patients such as age, gender, medical history, risk factors and laboratory results including complete blood count results were recorded. From the laboratory results obtained at admission to the emergency department within the first hour of application, immature granulocyte percentage (IG), white blood cell count (WBC), neutrophil, lymphocyte, platelet, hemoglobin, glucose, C-reactive protein (CRP) were measured by Sysmex XN-1000 modular system (Sysmex, Kobe, Japan). IG is a routine hemogram parameter and was obtained from whole blood analysis. The 1-month mortality of the patients were followed-up.

\section{Statistical analysis}

Statistical analyses in our study were performed using the SPSS 21.0 package program (SPSS Inc., Chicago, IL). Continuous variables were expressed as mean \pm standard deviation, and categorical variables as number (\%). Independent t-test was used for comparing the distribution of normally distributed parameters; Mann-Whitney U test was used for those who did not show normal distribution; in addition, categorical data were evaluated with the chi-square test. The optimum cut-off value of IG in mortality prediction in patients with AIS was evaluated by receiver operating characteristic (ROC) analysis and analysis of variables that may affect mortality by logistic regression analysis. Statistical significance was defined as a $\mathrm{p}$ value less than 0.05 .

\section{RESULTS}

In our study, 172 patients who met the inclusion criteria were included. Of these patients, 94
$(54.7 \%)$ were male, and $78(45.3 \%)$ were female. The mean age of the patients was calculated as $69.19 \pm 14.34$ years. Hypertension, coronary heart disease and diabetes were the most common risk factors. The mean GCS value of the patients were calculated as 12 (6), the mean NIHSS score was calculated as $10(4)$. The mean WBC value was $11.26 \pm 4.6910^{3} / \mathrm{mm}^{3}$. Mean CRP and IG values were measured as $62.12 \pm 30.94 \mathrm{mg} / \mathrm{land} 0.8 \pm$ $0.1 \%$, respectively. The clinical characteristics of the patients with AIS were listed in Table 1.

Table 1: The clinical characteristics of patients with acute ischemic stroke

\begin{tabular}{|c|c|}
\hline Variables & $\begin{array}{l}\text { Total } \\
(n=172)\end{array}$ \\
\hline Age (years) & $69.19 \pm 14.34$ \\
\hline Male gender n(\%) & $94(54.7)$ \\
\hline \multicolumn{2}{|l|}{ Clinical history } \\
\hline Hypertension & $91(52.9)$ \\
\hline Diabetes mellitus & $44(25.6)$ \\
\hline Hyperlipidemia & $15(8.7)$ \\
\hline Atrial fibrillation & $31(18.0)$ \\
\hline Coronary artery disease & $48(27.9)$ \\
\hline Prior stroke/TIA & $4(2.3)$ \\
\hline Glasgow Coma Scale & $12.84 \pm 2.76$ \\
\hline NIHSS & $10.82 \pm 5.24$ \\
\hline \multicolumn{2}{|l|}{ Pre stroke medications } \\
\hline Antiplatelet agents & $91(52.9)$ \\
\hline Oral anticoagulants & $38(22.1)$ \\
\hline Statins & $29(16.9)$ \\
\hline Antihypertensive drugs & $107(62.2)$ \\
\hline \multicolumn{2}{|l|}{ Laboratory features } \\
\hline White blood cell $\left(\mathrm{mm}^{3}\right)$ & $11.26 \pm 4.69$ \\
\hline Neutrophil $\left(10^{3} / \mathrm{mm}^{3}\right)$ & $8.61 \pm 4.56$ \\
\hline Lymhocyte $\left(10^{3} / \mathrm{mm}^{3}\right)$ & $1.71 \pm 01.04$ \\
\hline Hemoglobin (g/dL) & $12.21 \pm 2.31$ \\
\hline Platelet $\left(10^{3} / \mathrm{mm}^{3}\right)$ & $252.86 \pm 108.31$ \\
\hline Glucose (mg/dl) & $142.36 \pm 56.24$ \\
\hline NLR & $7.22 \pm 5.73$ \\
\hline PLR & $193.81 \pm 150.91$ \\
\hline CRP (mg/dl) & $62.12 \pm 30.94$ \\
\hline IG $(\%)$ & $0.8 \pm 0.1$ \\
\hline
\end{tabular}

TIA: Transient ischemic attack; NIHSS: The National Institutes of Health Stroke Scale; NLR: neutrophillymphocyte ratio; PLR: Platelet/lymphocyte ratio; CRP: C-reactive protein; IG: Immature granulocyte 
Patients were classified into two groups as low $(<0.6 \%)$ and high $(\geq 0.6 \%)$ by IG values. Of these patients, $98(56.9 \%)$ were in the low IG group, and $74(43.1 \%)$ of them were in the poor outcome group. According to laboratory parameters, compared to low IG group, patients in the high IG group had significantly higher WBC values $\left(12.45 \pm 3.3710^{3} / \mathrm{mm}^{3}\right.$ vs. $10.04 \pm$ $\left.3.610^{3} / \mathrm{mm}^{3} ; \mathrm{p}=0.001\right)$, higher neutrophil count $\left(9.3 \pm 2.5710^{3} / \mathrm{mm}^{3}\right.$ vs. $7.40 \pm 3.5510^{3} / \mathrm{mm}^{3} ; \mathrm{p}<$ $0.001)$, and higher glucose $(157.75 \pm 64.49 \mathrm{mg} /$ dlvs. $130.74 \pm 46.78 \mathrm{mg} / \mathrm{dl} ; \mathrm{p}<0.001)$ and higher $\mathrm{CRP}$ value $(89.25 \pm 11.41 \mathrm{mg} / \mathrm{l}$ vs. $41.42 \pm 8.07$ $\mathrm{mg} / \mathrm{l} ; \mathrm{p}<0.001)$. Grouping by stroke subtypes the percentage of large artery atherosclerosis was significantly higher in high IG group $(\mathrm{p}<0.001)$. In addition, hemoglobin values were found to be significantly lower in the high IG group compared to the low IG group $(11.41 \pm 2.32 \mathrm{~g} /$ dlvs. 12.79 $\pm 2.13 \mathrm{~g} / \mathrm{dl} ; \mathrm{p}<0.001)$ (Table 2). $7(17.9 \%)$ of mortality is pneumonia that develops within the first week, urinary system infection that develops after $3(7.7 \%)$ hospitalization, $20(51.3 \%)$ of them occurred due to cardiovascular complications (pulmonary embolism, arrhythmia, etc.) and 9 (23.1\%) patients for other reasons other than infectious.

In multivariate logistic regression analysis, blood glucose (1.006 (1.002-1.013), $\mathrm{p}=0.028)$, CRP (OR $1.005(1.001-1.009), \mathrm{p}=0.001)$ and IG (1.361 (1.194-1.966), $\mathrm{p}=0.014)$ were the strongest predictors of mortality in patients with AIS (Table 3). In addition, in the receiver operating characteristic (ROC) curve analysis, IG at the cut-off value of 1.3 was shown to predict mortality in patients with AIS with $80.5 \%$ sensitivity and $93.2 \%$ specificity (area under the curve: $0.71595 \%$ CI: 0.623-0.807, p <0.001). IG in predicting AIS mortality was much higher than other parameters [(AUC for ASPECTS: 0.712; sensitivity: 68.2\%; specificity: $80.7 \%$ ); [(AUC for CRP: 0.644 ; sensitivity: $60.5 \%$; specificity: 75.3\%)]. (Figure 1).

\section{DISCUSSION}

The results of our study are that IG is a simple, easily applicable and noninvasive prognosis marker in patients with AIS. Our results show that this 1.3 cut-off value can be used to predict mortality in patients with AIS.

The pathophysiological role of inflammation in AIS has been proven by studies. ${ }^{11}$ After ischemic stroke, the migration of immune system mediators and the release of cytokines take place, and this causes impairment in the permeability of the blood-brain barrier, which leads to brain edema and an increase in infarct volume and leads to neuronal damage. ${ }^{12}$ In recent studies, many non-invasive markers have been reported to be effective in poor prognosis and treatment in patients with AIS. ${ }^{13,14}$ In their study, Tokgöz et al. have found that increased infarct volume and NLR were significant for early mortality. ${ }^{15}$ In a study conducted by Martínez-Velilla et al. on geriatric patients with AIS, they have shown that red cell distribution width (RDW) was associated with mortality. ${ }^{16}$ In another study, they have shown that RDW was higher in patients who had a stroke and died on follow-up than those who survived, and that higher RDW values were associated with both cardiovascular mortality and all-cause mortality in stroke patients. ${ }^{17}$ Recent studies have shown the role of leukocyte in early plaque formation, and it has been reported that this may lead to AIS, and it has also been reported that the increase in WBC and its subtypes may be an independent risk factor. ${ }^{18,19}$ However, there is no publication examining the relationship of IG with patients with AIS.

IG is an early marker of bone marrow activation, which can be easily, and quickly measured from peripheral blood without the need for additional equipment, and in recent years provides valuable data for physicians to diagnose, or to examine patients in more detail. ${ }^{20}$ In recent studies, it has been reported that IG may be an indicator of inflammation in the early stages of many diseases. ${ }^{21}$ This new parameter has not been used sufficiently by many physicians, and its areas of use have not been clearly revealed. Studies have shown that increased IG values are associated with acute appendicitis, pancreatitis and cardiovascular system diseases. ${ }^{8,22,23}$ In a study conducted by Huang et al. on 1933 patients with acute pancreatitis, they have shown that increasing IG values predicted the risk of early ARDS development more effectively than known pancreatitis risk scores. ${ }^{24}$ Ayres et al. have reported in their study that for IG $<2.0,90 \%$ of the diagnosis of sepsis could be excluded, and it would be useful for early treatment initiation. ${ }^{10}$ Kong et al. have demonstrated that IG is an independent risk factor for 30-day mortality in patients undergoing angiography for ST-elevation myocardial infarction (STEMI) ${ }^{25}$ In another recent study, in patients with upper gastrointestinal bleeding admitted to the emergency room, IG > 1.0 at the time of admission was associated with an increased risk of 30-day mortality (OR, 40.9; 
Table 2: Clinical and laboratory findings in patients with low and high immature granulocyte

\begin{tabular}{|c|c|c|c|}
\hline Variables & $\begin{array}{l}\text { Low-IG group } \\
(<0.6, \mathbf{n}=98)\end{array}$ & $\begin{array}{l}\text { High -IG group } \\
(\geq 0.6, n=74)\end{array}$ & $P$ \\
\hline Age (years) & $66.72 \pm 15.57$ & $72.46 \pm 11.85$ & 0.022 \\
\hline Male gender n(\%) & $58(59.2)$ & $36(48.6)$ & 0.216 \\
\hline \multicolumn{4}{|l|}{ Clinical history } \\
\hline Hypertension & $49(50)$ & $42(56.8)$ & 0.425 \\
\hline Diabetes mellitus & $22(22.4)$ & $22(29.7)$ & 0.365 \\
\hline Hyperlipidemia & $10(10.2)$ & $5(6.8)$ & 0.529 \\
\hline Atrial fibrillation & $17(17.3)$ & $14(18.9)$ & 0.671 \\
\hline Coronary artery disease & $29(29.6)$ & $19(25.7)$ & 0.623 \\
\hline Prior stroke/TIA & $1(1)$ & $3(4.1)$ & 0.294 \\
\hline Glasgow Coma Scale & $13.06 \pm 2.7$ & $12.55 \pm 2.84$ & 0.225 \\
\hline NIHSS & $10.62 \pm 4.75$ & $11.13 \pm 6.05$ & 0.451 \\
\hline ASPECTS & $9(7-10)$ & $8(6-9)$ & 0.022 \\
\hline Subgroups according to etiology, n (\%) & & & $<0.001$ \\
\hline Large artery atherosclerosis & $35(35.7)$ & $37(50)$ & \\
\hline Cardioembolic causes & $32(32.7)$ & $24(32.4)$ & \\
\hline Lacunar infaret & $21(21.4)$ & $7(9.4)$ & \\
\hline Others & $10(10.2)$ & $6(8.2)$ & \\
\hline \multicolumn{4}{|l|}{ Pre stroke medications } \\
\hline Antiplatelet agents & $51(52)$ & $40(54.1)$ & 0.656 \\
\hline Oral anticoagulants & $21(21.4)$ & $17(23)$ & 0.675 \\
\hline Statins & $18(18.4)$ & $11(14.9)$ & 0.604 \\
\hline Antihypertensive drugs & $60(61.2)$ & $47(63.5)$ & 0.636 \\
\hline \multicolumn{4}{|l|}{ Laboratory features } \\
\hline White blood cell $\left(10^{3} / \mathrm{mm}^{3}\right)$ & $10.04 \pm 3.6$ & $12.45 \pm 3.37$ & 0.001 \\
\hline Neutrophil $\left(10^{3} / \mathrm{m} \mathrm{mm}^{3}\right)$ & $7.40 \pm 3.55$ & $9.3 \pm 2.57$ & $<0.001$ \\
\hline Lymhocyte $\left(10^{3} / \mathrm{m} \mathrm{mm}^{3}\right)$ & $1.81 \pm 0.19$ & $2.12 \pm 0.28$ & 0.352 \\
\hline Hemoglobin (g/L) & $12.79 \pm 2.13$ & $11.41 \pm 2.32$ & $<0.001$ \\
\hline Platelet $\left(10^{3} / \mathrm{mm}^{3}\right)$ & $238.81 \pm 81.36$ & $271.47 \pm 134.41$ & 0.146 \\
\hline Glucose (mg/dl) & $130.74 \pm 46.78$ & $157.75 \pm 64.49$ & $<0.001$ \\
\hline NLR & $6.56 \pm 5.26$ & $7.44 \pm 6.18$ & 0.527 \\
\hline PLR & $186.22 \pm 145.65$ & $195.72 \pm 143.34$ & 0.588 \\
\hline CRP (mg/dl) & $41.42 \pm 8.07$ & $89.25 \pm 11.41$ & $<0.001$ \\
\hline Therapy strategy, n (\%) & & & 0.455 \\
\hline Endovascular/thrombolytic & $21(21.4)$ & $18(24.3)$ & \\
\hline Anti-aggregant only & $77(78.6)$ & $56(75.6)$ & \\
\hline 30-day mortality [n (\%)] & $11(11.2)$ & $28(37.8)$ & $<0.001$ \\
\hline
\end{tabular}

TIA: Transient ischemic attack; NIHSS: The National Institutes of Health Stroke Scale; ASPECTS: the Alberta Stroke Program Early CT ScoreNLR: neutrophil-lymphocyte ratio; PLR: Platelet/lymphocyte ratio; CRP: C-reactive protein; IG: Immature granulocyte 
Table 3: Independent predictors of risk factors affected 30-day mortality in acute ischemic stroke

\begin{tabular}{llccc}
\hline & \multicolumn{2}{c}{ Univariate analysis } & \multicolumn{1}{c}{ Multivariate analysis } \\
\hline Variables & OR (95\% CI) & $p$ & OR (95\% CI) & $p$ \\
Age & $1.133(0.536-2.396)$ & 0.744 & $1.008(0.978-1.966)$ & 0.217 \\
Hypertension & $1.791(0.841-3.816)$ & 0.131 & $1.794(0.744-4.328)$ & 0.161 \\
Diabetes mellitus & $1.068(0.468-2.433)$ & 0.876 & $0.588(0.208-1.664)$ & 0.807 \\
Glucose $\geq \mathbf{1 0 0}$ & $1.017(1.001-1.014)$ & 0.017 & $1.006(1.002-1.013)$ & 0.028 \\
ASPECTS & $1.234(1.045-2.345)$ & 0.024 & $1.212(1.134-2.456)$ & 0.012 \\
Thrombolysis/ & $1.248(1.110-1.645)$ & 0.032 & $1.346(1.216-1.644)$ & 0.045 \\
thrombectomy & $1.589(1.021-2.456)$ & 0.003 & $1.254(1.116-2.216)$ & 0.001 \\
CRP $(\geq 3$ or $<3)$ & $1.245(1.145-2.648)$ & 0.018 & $1.312(1.214-2.632)$ & 0.024 \\
Infection $($ had vs had no) & $\mathbf{1}$ & $1.361(1.194-1.966)$ & 0.014 \\
IG\% $(\geq \mathbf{0 . 6}$ or $<\mathbf{0 . 6})$ & $1.487(1.027-2.152)$ & 0.035 & & \\
\hline
\end{tabular}

IGC: Immature granulocyte count; IG\%: Immature granulocyte percentage; CRP: C-reactive protein; ${ }^{1}$ : Infection development during hospitalization

95\% CI, 20.8-80.5; $\mathrm{p}<0.001){ }^{26}$ To the best of our knowledge, there were no studies in the literature examining the relationship between IG value and mortality in patients with AIS. In our study, there was a significant relationship between high IG value and 30-day mortality in patients with AIS.

Our study had some limitations. The first of these is that our study was conducted retrospectively as single-centered and with a relatively small number of patients. Another important limitation of ours is that the time from the onset of the patient's symptom to the diagnosis of AIS and sampling was not measured, and that this can affect the parameters. Another limitation is that serial IG measurement and other factors that may affect mortality have not been revealed clearly. In addition, some inflammatory parameters such as tumor necrosis factor and interleukin-6

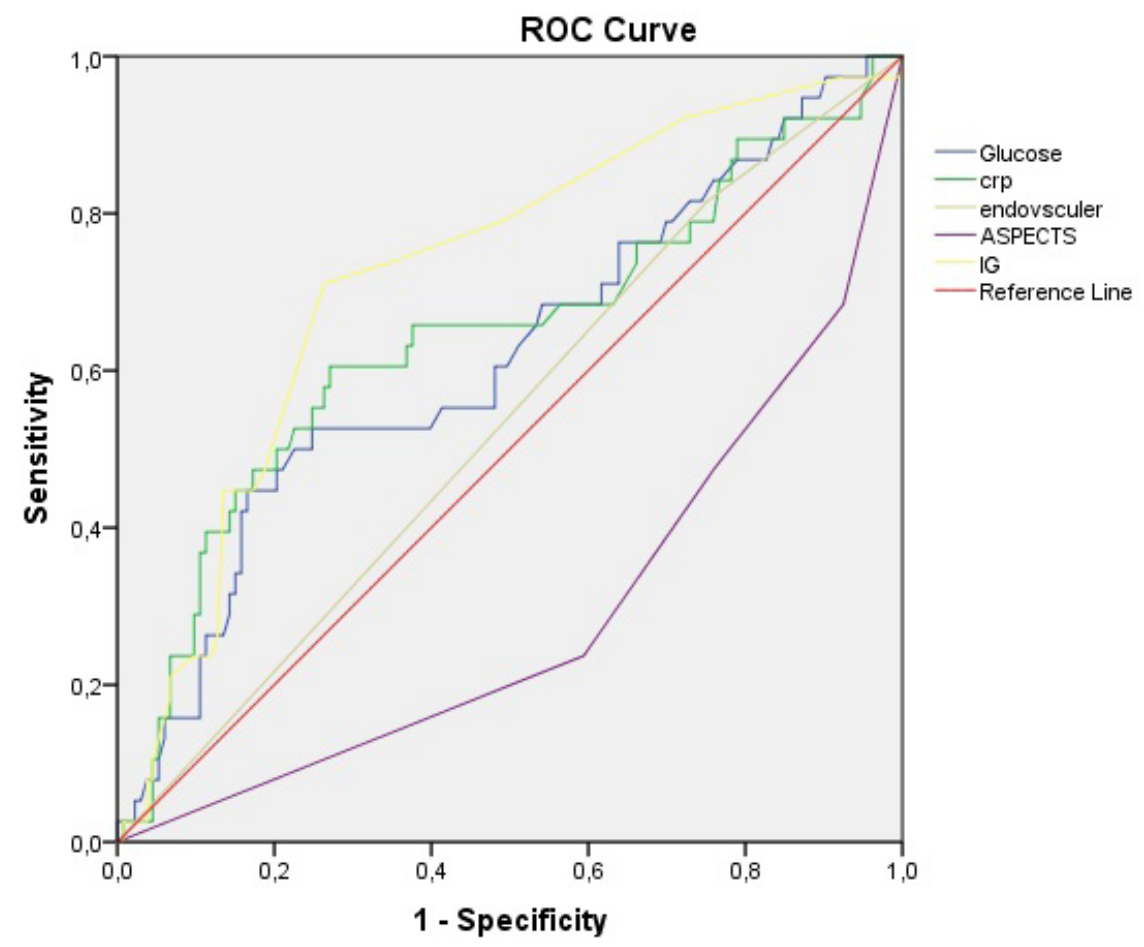

Figure 1. Receiver operating characteristic curve analysis of parameters in the discrimination between mortality 
levels could not be compared in our IG study. We think that conducting multi-centered prospective studies with a larger patient population in order to better analyze the data in our study will provide clearer results.

In conclusion, the results of our study showed that IG is a new and simple predictor to predict 30-day prognosis in patients with AIS.

\section{REFERENCES}

1. Campbell BC, De Silva DA, Macleod MR, et al. Ischaemic stroke. Nat Rev Dis Primers 2019;5(1):122.

2. Şensöz NP, Börü ÜT, Bölük C, et al. Stroke epidemiology in Karabük city Turkey: Community based study. Eneurologicalsci 2018;10:12-5.

3. Li X, Lin S, Chen X, et al. The prognostic value of serum cytokines in patients with acute ischemic stroke. Aging Dis 2019;10(3):544.

4. Worthmann H, Tryc A, Deb M, et al. Linking infection and inflammation in acute ischemic stroke. Ann NY Acad Sci 2010;1207:116-22.

5. Ren H, Liu X, Wang L, Gao Y. Lymphocyte-tomonocyte ratio: a novel predictor of the prognosis of acute ischemic stroke. J Stroke Cerebrovasc Dis 2017;26(11):2595-602.

6. Jin P, Li X, Chen J, et al. Platelet-to-neutrophil ratio is a prognostic marker for 90-days outcome in acute ischemic stroke. J Clin Neurol 2019;63:110-5.

7. Wang L, Song Q, Wang C, et al. Neutrophil to lymphocyte ratio predicts poor outcomes after acute ischemic stroke: a cohort study and systematic review. J Neurol Sci 2019;406:116445.

8. Korkut M, Bedel C, Selvi F. Are immature granulocytes and derivatives early predictors of acute appendicitis and acute complicated appendicitis in adults? Formos J Surg 2020;53(4):123.

9. Karakulak S, Narcı H, Ayrık C, Erdoğan S, Üçbilek E. The prognostic value of immature granulocyte in patients with acute pancreatitis. Am J Emerg Med 2020; 44:203-7.

10. Ayres LS, Sgnaolin V, Munhoz TP. Immature granulocytes index as early marker of sepsis. Int $J$ Lab Hematol 2019;41(3):392-6.

11. Kim JY, Kawabori M, Yenari MA. Innate inflammatory responses in stroke: mechanisms and potential therapeutic targets. Curr Med Chem 2014;21:2076-97.

12. Petrovic-Djergovic D, Goonewardena SN, Pinsky DJ. Inflammatory disequilibrium in stroke. Circ Res 2016;119:142-58.

13. Qun S, Tang Y, Sun J, et al. Neutrophil-to-lymphocyte ratio predicts 3-month outcome of acute ischemic stroke. Neurotox Res 2017;31(3):444-52.

14. Maestrini I, Strbian D, Gautier S, et al. Higher neutrophil counts before thrombolysis for cerebral ischemia predict worse outcomes. Neurology 2015;85:1408-16.

15. Tokgoz S, Keskin S, Kayrak M, Seyithanoglu A, Ogmegul A. Is neutrophil/lymphocyte ratio predict to short-term mortality in acute cerebral infarct independently from infarct volume? J Stroke Cerebrovasc Dis 2014;23:2163-8.

16. Martínez-Velilla N, Ibáñez B, Cambra K, AlonsoRenedo J. Red blood cell distribution width, multimorbidity, and the risk of death in hospitalized older patients. Age 2012;34:717-23.

17. Ani C, Ovbiagele B. Elevated red blood cell distribution width predicts mortality in persons with known stroke. J Neurol Sci 2009;277(1-2):103-8.

18. Qu X, Shi J, Cao Y, Zhang M, Xu J. Prognostic value of white blood cell counts and c-reactive protein in acute ischemic stroke patients after intravenous thrombolysis. Curr Neurovasc Res 2018;15(1):10-7.

19. Zheng X, Zeng N, Wang A, et al. Prognostic value of white blood cell in acute ischemic stroke patients. Curr Neurovasc Res 2018;15(2):151-7.

20. Senthilnayagam B, Kumar T, Sukumaran J, Rao K. Automated measurement of immature granulocytes: performance characteristics and utility in routine clinical practice. Patholog Res Int 2012;2012: 483670.

21. Park JH, Byeon HJ, Lee KH, et al. Delta neutrophil index (DNI) as a novel diagnostic and prognostic marker of infection: a systematic review and metaanalysis. $J$ Inflamm Res 2017;66:863-70.

22. Bedel C, Korkut M, Aksoy F, Kus G. Usefulness of immature granulocytes to predict high coronary SYNTAX Score in acute coronary syndrome; a Crosssectional study: Usefulness of immature granulocytes to predict high coronary syntax score. Arch Acad Emerg Med 2020;8(1):e73.

23. Ünal Y. A new and early marker in the diagnosis of acute complicated appendicitis: immature granulocytes. Ulus Travma Acil Cerrahi Derg 2018;24:434-9.

24. Huang Y, Xiao J, Cai T, et al. Immature granulocytes: a novel biomarker of acute respiratory distress syndrome in patients with acute pancreatitis. J Crit Care 2019;50:303-8.

25. Kong T, Park YS, Lee HS. The delta neutrophil index predicts development of multiple organ dysfunction syndrome and 30-day mortality in trauma patients admitted to an intensive care unit: a retrospective analysis. Sci Rep 2018;8:17515.

26. Kong T, In S, Park YS, et al. Usefulness of the Delta Neutrophil Index to Predict 30-Day Mortality in Patients with Upper Gastrointestinal Bleeding. Shock (Augusta, Ga) 2017;48:427-35. 